\title{
New insights into pathways of the accessory nerve and transverse cervical artery for distal selective accessory nerve blockade
}

\author{
Yanguk Heo ${ }^{1,2}$, Namju Cho ${ }^{1}$, Hyunho Cho ${ }^{2,3}$, Hyung-Sun Won ${ }^{1,2}$, Miyoung Yang ${ }^{1,2}$, and Yeon-Dong Kim ${ }^{2,3,4}$ \\ 'Department of Anatomy, Wonkwang University School of Medicine, Iksan, Korea \\ 'Jesaeng-Euise Clinical Anatomy Center, Wonkwang University School of Medicine, Iksan, Korea \\ ${ }^{3}$ Department of Anesthesiology and Pain Medicine, Wonkwang University Hospital, Wonkwang University School of Medicine, Iksan, Korea \\ ${ }^{4}$ Wonkwang Institute of Science, Wonkwang University School of Medicine, Iksan, Korea
}

Received May 31, 2019

Revised September 6, 2019

Accepted September 10, 2019

\section{Correspondence}

Yeon-Dong Kim

Department of Anesthesiology and Pain Medicine, Wonkwang University Hospital, Wonkwang University School of Medicine, 895 Muwangro, Iksan 54538, Korea Tel: +82-63-859-1562

Fax: +82-63-857-5472

E-mail: kydpain@hanmail.net
Background: The aim of this study was to clarify the topographical relationship between the accessory nerve (AN) and transverse cervical artery (TCA) to provide safe and convenient injection points for AN blockade.

Methods: This study included 21 and 30 shoulders of 14 embalmed Korean adult cadavers and 15 patients, respectively, for dissection and ultrasound (US) examination.

Results: The courses of the TCA and AN in the scapular region were classified into four types based on their positional relationships. Type A indicated the nerve that was medial to the artery and ran parallel without changing its location (38\%). In type B (38\%), the nerve was lateral to the artery and ran parallel without changing its location. In type C (19\%), the nerve or artery traversed each other only once during the whole course. In type D (5\%), the nerve or artery traversed each other more than twice forming a twist. At the levels of lines I-IV, the nerve was relatively close to the artery (approximately $10 \mathrm{~mm}$ ). TCAs were observed in all specimens around the superior angle of the scapula at the level of line II, whereas they were not found below line VI. In US images of the patients, the TCA was commonly observed at the level of line II (93.3\%) where all ANs and TCAs were observed in cadaveric dissection.

Conclusions: The results expand the current knowledge of the relation between the AN and TCA, and provide helpful information for selective diagnostic nerve blocks in the scapular region.

Key Words: Accessory Nerve; Diagnosis; Injections; Scapula; Scapular Region; Selective Nerve Blockade; Transverse Cervical Artery; Ultrasound

\section{INTRODUCTION}

The external branch of the accessory nerve (AN) passes the carotid and posterior triangles of the neck, and innervates the sternocleidomastoid (SCM) and trapezius muscles (Tz) [1]. This nerve could be entrapped or injured due to whip- lash injury with the head turned or other causes, or adhesion following surgery involving other peripheral nerves $[2,3]$. The AN, while innervating the muscle, has multiple connections to other nerves including the occipital, cervical, and brachial plexus, and thus, can be involved in various signs and symptoms related to pain $[4,5]$. Moreover, (c) This is an open-access article distributed under the terms of the Creative Commons Attribution Non-Commercial License (http://creativecommons.org/licenses/by-nc/4.0/), which permits unrestricted non-commercial use, distribution, and reproduction in any medium, provided the original work is properly cited.

(C) The Korean Pain Society, 2020
Author contributions: Yanguk Heo: Investigation, Writing/manuscript preparation; Namju Cho: Investigation; Hyunho Cho: Investigation; HyungSun Won: Writing/manuscript preparation; Miyoung Yang: Writing/manuscript preparation; Yeon-Dong Kim: Investigation, Writing/manuscript preparation, Study conception. 
secondary myofascial pain caused by prolonged nerve entrapment could be difficult to diagnose [6], as there are different nerves innervating each muscle of the shoulder and neck. When the pain occurs in the neck and shoulder regions, differential diagnosis is mainly based on history (surgery, trauma, or neurologic disorders) and physical examination, which is often misinterpreted.

Adequate pain relief following selective blockade by assessing the innervated area can be regarded as a valuable diagnostic tool for AN entrapment. Two methods of approaching the nerve for the nerve blockade have been currently introduced. One is to approach towards the styloid process of the temporal bone using a landmark-guided technique, nerve stimulator, or fluoroscope, and the other is to approach through the posterior triangle of the neck using ultrasound (US) [7-9]. However, it is not easy to manage the AN entrapment through precise blockade with the aforementioned methods, because of the distribution of other significant nerves such as the phrenic nerve and cervical sympathetic trunk at the injection site [10]. Furthermore, since the patient's symptoms caused by AN entrapment will be considered during selective AN blockade, a more distal and posterior approach method for those symptoms associated only with the Tz would be safer and more reasonable for effective management and diagnosis. However, the AN can be often mistakenly considered as a fibrous tissue on US image because of its small diameter $(<1-2 \mathrm{~mm})$ and oblique course at the intermuscular planes [11]. This invisibility gets worse under the Tz in the scapular region raising the need for a new landmark for nerve blockade. Thus, this study was performed to clarify the topographical relationships between the AN and trans-

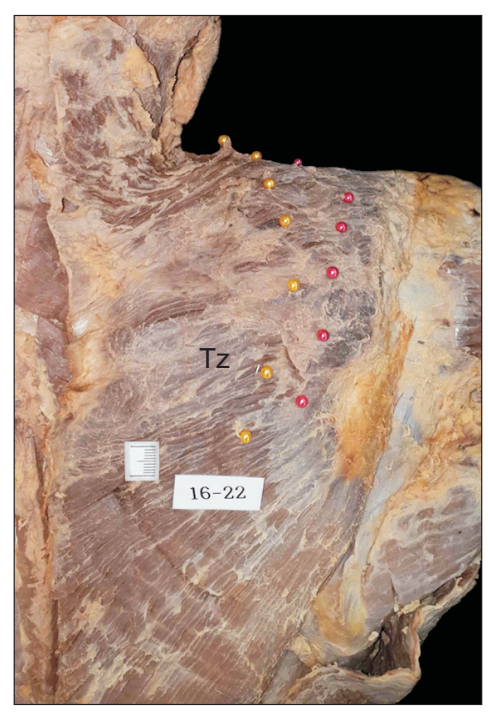

Fig. 1. Marking method showing the course of the accessory nerve (yellow colored head pins) and transverse cervical artery (red colored head pins). Tz: trapezius muscles. verse cervical artery (TCA) that pass under the Tz in the scapular region by comparing the anatomical dissectionbased analysis and US images, in order to suggest newer, safer, and more convenient injection points for selective AN blockade.

\section{MATERIALS AND METHODS}

This study was approved by the Institutional Review Board of Wonkwnag University (IRB ID No. WKIRB-201905BM-033).

\section{Cadaveric observation}

Twenty-one shoulders of 14 embalmed Korean adult cadavers ( 7 males and 7 females; mean age, $83.1 \mathrm{yr}$ at death; range, 58-95 yr) were dissected. All cadavers were stored at the Jesaeng-Euise Institute of Wonkwang University School of Medicine, and were qualified as materials for use in education and research according to national law.

None of the cadavers exhibited any evidence of gross pathology, previous surgical procedures, or traumatic lesions in the target region. After removing the skin and subcutaneous tissue, the $\mathrm{Tz}$ was exposed. This muscle was cut at its origin site and reflected laterally with careful confirmation of the running course of the AN and TCA, without any change in their locations. The AN and TCA were then marked with colored head pins at the levels of the defined

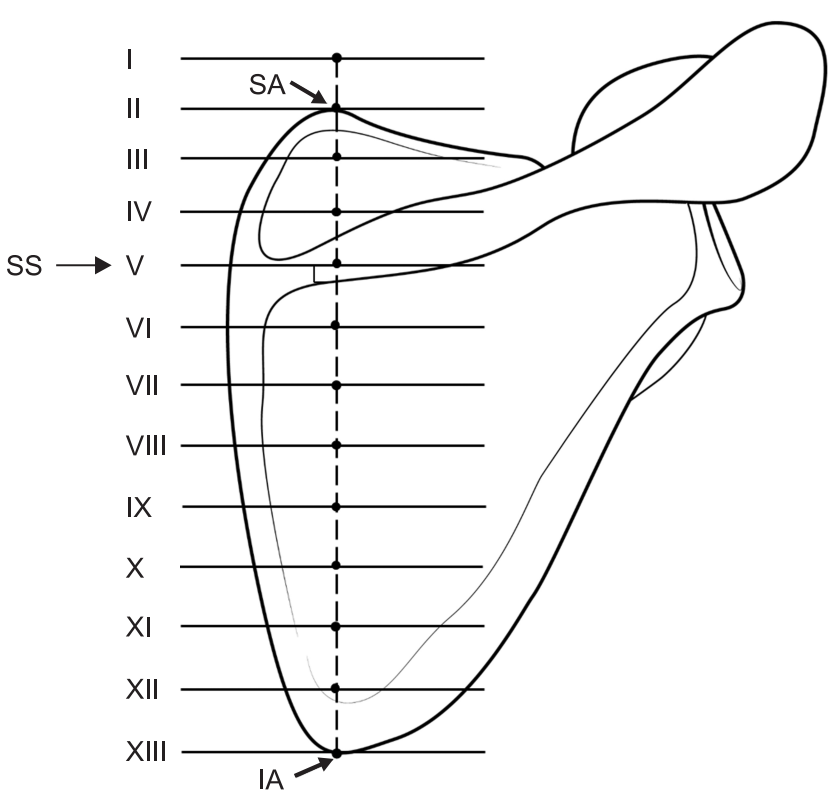

Fig. 2. Lines and points defined for analyzing the positional relationship between the spinal accessory nerve and reference line connecting the superior and inferior angles of the scapula. SA: superior angle of scapula, SS: spine of scapula, IA: inferior angle of scapula. 
points (Fig. 1).

\section{Measurement}

The authors first defined a line and some points to analyze the courses of the AN and TCA in their anatomical position (Fig. 2). The reference line was the line connecting the superior and inferior angles of the scapula. The scapula was divided into two sections based on the scapular spine. The upper section was divided into three equal parts through its whole length and an additional point was marked farthest away on each part on an imaginary extended line from the reference line, while the lower section was divided into eight equal parts. Locations and distances of both the AN and TCA were measured on each point of the perpendicular lines from the 13 defined points using a digital caliper (Mitutoyo, Tokyo, Japan). Except where the number of samples was less than 5 , all distances are presented as mean \pm standard deviation values.

\section{Patients US observation}

The study included examination of 30 shoulders of 15 patients ( 6 males and 9 females; mean age, $51.3 \mathrm{yr}$; range, 24$77 \mathrm{yr}$ ). Informed consent was obtained from the patient. None of the patients had a history of trauma, surgery, or structural pathology in the target region. The transducer of the US (Linear probe 12-5 MHz, Samsung HS 60; Samsung Medison, Seoul, Korea) was placed transversely at the levels of the significant lines based on the data from the cadaveric dissection.

\section{RESULTS}

In a cadaveric study, the running patterns of the TCA and AN below the Tz were classified into four types based on their positional relationships. If the nerve was to the artery and ran parallel to it without changing its location along the whole course, it was classified as type A (38\%). In type
$\mathrm{B}(38 \%)$, the nerve was lateral to the artery and ran parallel without changing its location. In type C (19\%), the nerve or artery traversed each other only once during the whole course, whereas in type D (5\%), the nerve or artery traversed each other more than twice, forming a twist.

The positional relationship and distance between the TCA and AN, at the level of each defined line were analyzed (Table 1). The nerve was medial and lateral to the artery in $37 \%$ and $63 \%$ cases, respectively, at the level of line I, and in $48 \%$ and $52 \%$ cases at level of line II, respectively, exhibiting a higher lateral tendency. On the other hand, the nerve was medial and lateral to the artery in $61 \%$ and $39 \%$ cases, respectively, at the level of line III, and in $56 \%$ and $44 \%$ cases at the level of line IV, respectively, exhibiting a higher medial tendency. The nerves were located approximately within $9.5 \mathrm{~mm}$ medial and $7.5 \mathrm{~mm}$ lateral to the artery above line $\mathrm{V}$.

The relationships between the TCA and the reference line and the AN and the reference line were analyzed (Fig. 3). At the levels of lines I and II, most of the arteries were lateral to the reference line, with a frequency of $94.7 \%$ (line I) and $80.9 \%$ (line II). The artery was medial and lateral to the reference line in $38.9 \%$ and $61.1 \%$ cases at the level of line III, respectively, and in $54.5 \%$ and $45.5 \%$ cases at the level of line IV, respectively. The arteries were located approximately within $16.2 \mathrm{~mm}$ medial and $16.5 \mathrm{~mm}$ lateral to the reference line above line V. At the levels of lines I and II, most of the nerves were lateral to the reference line with a frequency of $89.5 \%$ (line I) and $76.2 \%$ (line II). At the level of line III, the frequency of the nerve being medial or lateral to the medial border of the scapula was similar. At the level of line IV, most of the nerves were medial to the reference line. At the levels of lines V and VI, all the nerves were medial to the reference line. Below line VI, the number of nerves identified by the naked eye was obviously reduced. The nerves were located approximately within $11.6 \mathrm{~mm}$ medial and $18.8 \mathrm{~mm}$ lateral to the medial border of the scapula above line $\mathrm{V}$.

In the analysis of the US images of the 30 shoulders of living patients, the TCA could be identified in the patients

Table 1. Measurement of Distance between the Accessory Nerve and Transverse Cervical Artery (TCA) at Each Line (Lines I-VI) of the Cadavers

\begin{tabular}{|c|c|c|c|c|c|}
\hline \multirow{2}{*}{ Line } & \multicolumn{2}{|c|}{ Medial to the TCA } & \multicolumn{2}{|c|}{ Lateral to the TCA } & \multirow{2}{*}{ Total } \\
\hline & Distance (mm) & Specimens & Distance (mm) & Specimens & \\
\hline I & $5.8 \pm 3.7(0.6-9.5)$ & 7 & $5.6 \pm 4.3(1.0-14.1)$ & 12 & 19 \\
\hline ॥ & $8.6 \pm 6.6(1.0-20.5)$ & 10 & $4.1 \pm 2.7(0.3-9.5)$ & 11 & 21 \\
\hline III & $6.1 \pm 5.1(0.3-15.3)$ & 11 & $7.5 \pm 6.1(1.7-18.1)$ & 7 & 18 \\
\hline IV & $9.5 \pm 4.7(4.0-16.5)$ & 5 & $6.4 \pm 4.7(3.1-13.2)$ & 4 & 9 \\
\hline V & $9.0 \pm 6.5(2.3-15.3)$ & 3 & 7 & 1 & 4 \\
\hline VI & 9.2 & 1 & 8.8 & 1 & 2 \\
\hline
\end{tabular}

Values are presented as mean \pm standard deviation (range) or number only. 

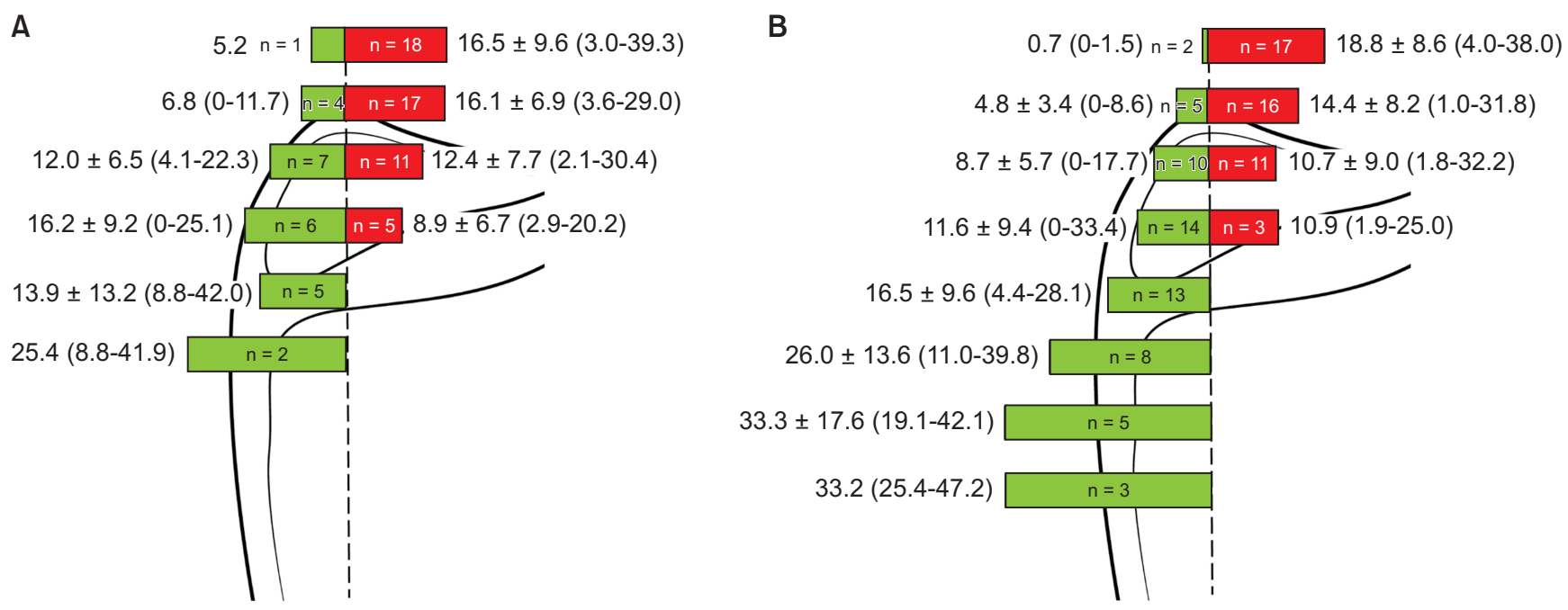

Fig. 3. Frequency and distance of the transverse cervical artery (A) and accessory nerve (B) at the level of each line of the cadavers (green and red colored bars indicate the structures passing toward the medial and lateral sides of the reference line connecting the superior and inferior angles of the scapula, respectively).
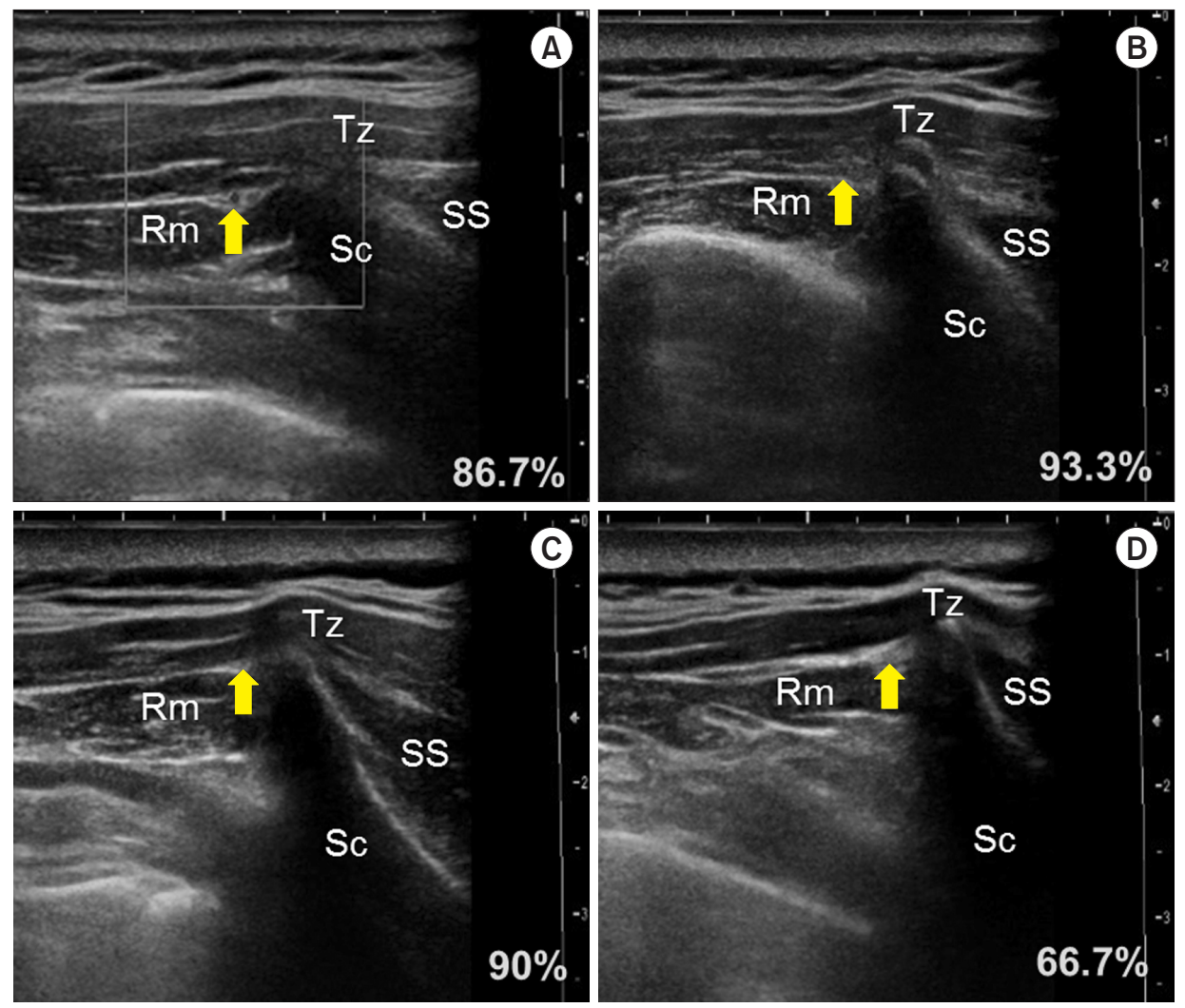

Fig. 4. Ultrasound (US) images and frequency of the detected transverse cervical artery; In the panels A-D show US images of the same patient at the lines I-IV, respectively. Tz: trapezius muscle, Rm: rhomboid minor muscle, Sc: scapula, SS: supraspinatus muscle.

by the US Doppler image showing its pulsation. In a previous cadaveric study, the number of arteries identified by the naked eye was obviously reduced below line IV. US examination, thus, was performed above line V. As a result, the artery was seen at line I in $86.7 \%(n=26)$, line II in 93.3\% ( $\mathrm{n}=28)$, line III in $90 \%(\mathrm{n}=27)$, and line IV in $66.7 \%$ $(n=20)$ (Fig. 4). In all cases, the TCA was observed, at least, on more than one line.

\section{DISCUSSION}

The AN entrapment, which is the compression of the external branch of the AN, can be theoretically associated with muscle atrophy, because this nerve is a purely motor nerve innervating the SCM and Tz [1]. This could lead to limited and sustained shoulder abduction, lateral winged scapula, or secondary painful shoulder syndrome caused by a 
traction injury of the brachial plexus in the unsupported shoulder [12]. Myofascial release, trigger point injection, nerve blockade, neurolysis, radiofrequency denervation, and surgery are common modes of management for AN entrapment [2]. Among these therapeutic interventions, selective AN blockade has been known to be a useful diagnostic tool that relaxes the Tz and reduces pain [9].

There are two approach methods for nerve blockade in managing AN entrapment. One is to approach towards the styloid process of the temporal bone [13,14]. Unfortunately, this approach has been proven to cause asystole due to stimulation of the vagus nerve [15] and is associated with the risk of intravascular injection due to abundant nearby blood vessels [16]. The other method is to approach through the posterior triangle of the neck [9]. However, it is not easy to block the AN selectively through this region, because of the presence of many other nerves including the cutaneous nerves arising from the cervical plexus, the phrenic nerve, and the trunk or root of the brachial plexus [17]. When the AN entrapment affects only the Tz, the aforementioned methods could be an overtreatment if the relationship with the AN has not been specified. It is also reported that chronic compartment syndromes of the Tz can cause entrapment of the AN and have a clinical presentation that closely matches the pattern of symptoms observed among patients with chronic whiplash [18].

Therefore, the present study introduces a safer and more reasonable nerve blockade with a new posterior approach for managing and diagnosing this kind of AN entrapment. The AN is known to give off one or more small branches to the upper Tz before coursing ventral to the muscle, and finally dividing into terminal branches that supply the middle and lower Tz. Moreover, the TCA is known to supply the upper region of the $\mathrm{Tz}[19,20]$.

The authors suggest two important points for facilitating a selective AN block based on the anatomical confirmation of the running course of the AN and TCA. First, injections with local anesthetics may be administered above line $\mathrm{V}$, and approximately $10 \mathrm{~mm}$ around the TCA for successful blockade. The TCA was detected with high frequency at the levels of the lines I-IV, and the nerve was located approximately within the region $9.5 \mathrm{~mm}$ medial and $7.5 \mathrm{~mm}$ lateral to the TCA at these levels. Second, the superior angle of the scapula can be regarded as a useful landmark for both blind and US-guided techniques. At the level of line II, the AN and TCA were observed in $100 \%$ of the cases of cadaveric dissection (Table 1), and all the ANs were close to the superior angle of the scapula. The results from US examinations also support the above recommendations. During US scanning, the TCA was most commonly observed at the level of line II ( $93.3 \%$ of the cases). Based on the aforementioned points, physicians who want to adapt

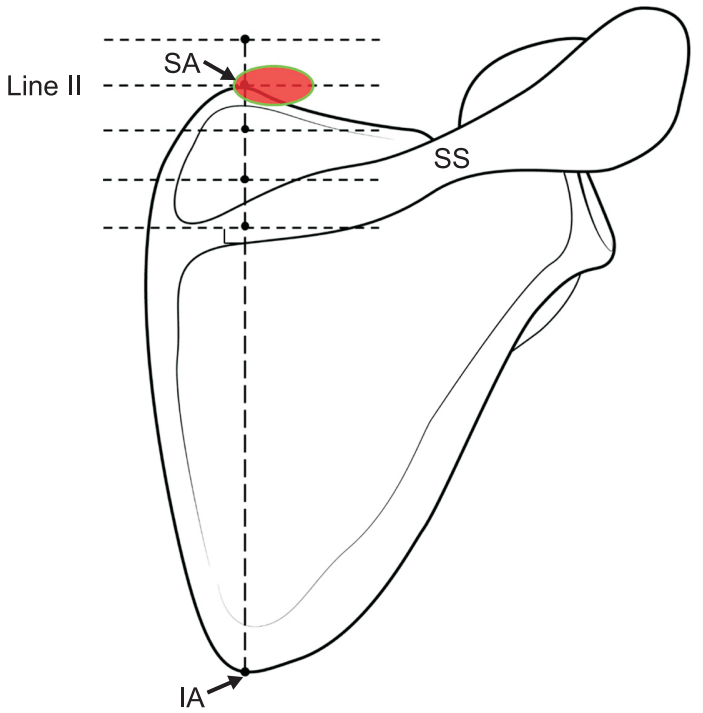

Fig. 5. Recommendable point under ultrasound guided or blind injection technique. SA: superior angle of scapula, SS: spine of scapula, IA: inferior angle of scapula.

the distal-posterior approach more easily must try to identify the TCA using US at the level of line II (the level of the superior angle of the scapula). When a physician succeeds in locating the TCA using the Doppler image, local anesthetic injection must be administered at approximately 10 $\mathrm{mm}$ around the TCA. In some cases, detecting the artery could be difficult depending on the US technician's proficiency or the patient's physiological conditions, such as the presence of thin blood vessels or obesity. In this case, the AN blockade can also be performed through a simple injection below the $\mathrm{Tz}$, around the superior angle of the scapula, considering the area medial $4.8 \mathrm{~mm}$ to lateral 14.4 mm (Fig. 5).

In conclusion, the results of this study provided appropriate and useful suggestions about the distal posterior approach using the US-guided or blind technique in the clinical field. However, this study had a limitation that it could not suggest the accurate success rate or optimal quantity of local anesthetics to be used for AN blockade in various regions. This limitation could be attributable to the observational and comparative nature of this study, and the limited number of cadaveric dissections performed and patients undergoing US examination. This can be resolved in future studies with cadaveric studies using dye injection or clinical trials based on the results of this study.

\section{ACKNOWLEDGMENTS}

The authors would like to thank Mr. Seong-In John at Setopito for preparing the figures. The authors thank the body donors and their families, as it would have been impos- 
sible for us to complete this study without their generous donation.

\section{CONFLICT OF INTEREST}

No potential conflict of interest relevant to this article was reported.

\section{FUNDING}

This study was supported by Wonkwang University in 2018.

\section{ORCID}

Yanguk Heo, https://orcid.org/0000-0003-0702-1288

Namju Cho, https://orcid.org/0000-0003-3877-7803

Hyunho Cho, https://orcid.org/0000-0003-2117-4280

Hyung-Sun Won, https://orcid.org/0000-0001-6084-6698

Miyoung Yang, https://orcid.org/0000-0002-4748-6007

Yeon-Dong Kim, https://orcid.org/0000-0003-0404-2657

\section{REFERENCES}

1. Shiozaki K, Abe S, Agematsu H, Mitarashi S, Sakiyama K, Hashimoto $\mathrm{M}$, et al. Anatomical study of accessory nerve innervation relating to functional neck dissection. J Oral Maxillofac Surg 2007; 65: 22-9.

2. Nystrom NA, Champagne LP, Freeman M, Blix E. Surgical fasciectomy of the trapezius muscle combined with neurolysis of the Spinal accessory nerve; results and long-term follow-up in 30 consecutive cases of refractory chronic whiplash syndrome. J Brachial Plex Peripher Nerve Inj 2010; 5: 7.

3. Chandawarkar RY, Cervino AL, Pennington GA. Management of iatrogenic injury to the spinal accessory nerve. Plast Reconstr Surg 2003; 111: 611-7.

4. Cappiello J, Piazza C, Giudice M, De Maria G, Nicolai P. Shoulder disability after different selective neck dissections (levels II-IV versus levels II-V): a comparative study. Laryngoscope 2005; 115: 259-63.

5. Restrepo CE, Tubbs RS, Spinner RJ. Expanding what is known of the anatomy of the spinal accessory nerve. Clin Anat 2015; 28: 467-71.

6. Bremner-Smith AT, Unwin AJ, Williams WW. Sensory path- ways in the spinal accessory nerve. J Bone Joint Surg Br 1999; 81:226-8.

7. Bodner G, Harpf C, Gardetto A, Kovacs P, Gruber H, Peer S, et al. Ultrasonography of the accessory nerve: normal and pathologic findings in cadavers and patients with iatrogenic accessory nerve palsy. J Ultrasound Med 2002; 21: 1159-63.

8. Kessler J, Gray AT. Course of the spinal accessory nerve relative to the brachial plexus. Reg Anesth Pain Med 2007; 32: 174-6.

9. Townsley P, Ravenscroft A, Bedforth N. Ultrasound-guided spinal accessory nerve blockade in the diagnosis and management of trapezius muscle-related myofascial pain. Anaesthesia 2011; 66: 386-9.

10. Canella C, Demondion X, Delebarre A, Moraux A, Cotten H, Cotten A. Anatomical study of phrenic nerve using ultrasound. Eur Radiol 2010; 20: 659-65.

11. Li AE, Greditzer HG 4th, Melisaratos DP, Wolfe SW, Feinberg JH, Sneag DB. MRI findings of spinal accessory neuropathy. Clin Radiol 2016; 71: 316-20.

12. Cappiello J, Piazza C, Nicolai P. The spinal accessory nerve in head and neck surgery. Curr Opin Otolaryngol Head Neck Surg 2007; 15: 107-11.

13. Choi $\mathrm{CH}$, Choi JH, Sung CH. Positive effects of local anesthetic nerve blocks for a patient with newly developed left side spasmodic torticollis after surgical intervention of right side spasmodic torticollis - a case report. Korean J Pain 2007; 20: 246-50.

14. Ramamurthy S, Akkineni SR, Winnie AP. A simple technic for block of the spinal accessory nerve. Anesth Analg 1978; 57: 591-3.

15. Scott PV. Asystole from tetanic stimulation of the accessory nerve. Anaesthesia 1996; 51: 1148-50.

16. Waldman SD. Atlas of interventional pain management. 3rd ed. Philadelphia, Saunders. 2009, p 103.

17. Symes A, Ellis H. Variations in the surface anatomy of the spinal accessory nerve in the posterior triangle. Surg Radiol Anat 2005; 27 : 404-8.

18. Hagert CG, Christenson JT. Hyperpressure in the trapezius muscle associated with fibrosis. Acta Orthop Scand 1990; 61: 263-5.

19. Jobe CM, Kropp WE, Wood VE. Spinal accessory nerve in a trapezius-splitting surgical approach. J Shoulder Elbow Surg 1996; 5: 206-8.

20. Ogawa R, Murakami M, Vinh VQ, Hyakusoku H. Clinical and anatomical study of superficial cervical artery flaps: retrospective study of reconstructions with 41 flaps and the feasibility of harvesting them as perforator flaps. Plast Reconstr Surg 2006; 118: 95-101. 